\title{
Mental health and suicide in former professional soccer players
}

\author{
Emma R Russell, ${ }^{1}$ Thomas McCabe, ${ }^{2}$ Daniel F Mackay, ${ }^{3}$ Katy Stewart, ${ }^{4,5}$ \\ John A MacLean, ${ }^{4,5}$ Jill P Pell, ${ }^{3}$ William Stewart (1) ${ }^{1,6}$
}

${ }^{1}$ Institute of Neuroscience and Psychology, University of Glasgow, Glasgow, UK ${ }^{2} S$ chool of Medicine, Dentistry and Nursing, University of Glasgow, Glasgow, UK ${ }^{3}$ Institute of Health and Wellbeing, University of Glasgow, Glasgow, UK ${ }^{4}$ Institute of Cardiovascular and Medical Sciences, University of Glasgow, Glasgow, UK ${ }^{5}$ Hampden Sports Clinic, Hampden Park, Glasgow, UK ${ }^{6}$ Department of Neuropathology, NHS Greater Glasgow and Clyde, Glasgow, UK

\section{Correspondence to}

DrWilliam Stewart, Department of Neuropathology, NHS Greater Glasgow and Clyde, Glasgow G51 4TF, UK; william.stewart@ glasgow.ac.uk

Received 23 March 2020 Revised 6 May 2020

Accepted 17 June 2020 Published Online First 21 July 2020

\section{SLinked}

- http://dx.doi.org/10.1136/ jnnp-2020-323616

Check for updates

(C) Author(s) (or their employer(s)) 2020. No commercial re-use. See rights and permissions. Published by BMJ.

To cite: Russell ER, McCabe T, Mackay DF, et al. J Neurol Neurosurg Psychiatry

2020:91:1256-1260.

\section{ABSTRACT}

Introduction There is growing recognition of an association between contact sports participation and increased risk of neurodegenerative disease, including Alzheimer's disease and chronic traumatic encephalopathy. In addition to cognitive impairment, a range of mental health disorders and suicidality are proposed as diagnostic features of traumatic encephalopathy syndrome, the putative clinical syndrome associated with chronic traumatic encephalopathy. However, to date, epidemiological data on contact sport participation and mental health outcomes are limited. Methods For a cohort of former professional soccer players ( $n=7676$ ) with known high neurodegenerative mortality and their matched general population controls $(n=23028)$, data on mental health outcomes were obtained by individual-level record linkage to national electronic records of hospital admissions and death certification.

Results Compared with matched population controls, former professional soccer players showed lower risk of hospital admission for anxiety and stress related disorders, depression, drug use disorders, alcohol use disorders and bipolar and affective mood disorders. Among soccer players, there was no significant difference in risk of hospitalisation for mental health disorders between outfield players and goalkeepers. There was no significant difference in rate of death by suicide between soccer players and controls.

Conclusions Among a population of former professional soccer players with known high neurodegenerative disease mortality, hospital admissions for common mental health disorders were lower than population controls, with no difference in suicide. Our data provide support for the reappraisal of currently proposed diagnostic clinical criteria for traumatic encephalopathy syndrome, in particular the inclusion of mental health outcomes.

\section{INTRODUCTION}

There are growing concerns around the risk of common mental health disorders and suicide in contact sports athletes. ${ }^{12}$ In part, this is driven by autopsy studies of retired athletes from a range of sports describing the frequent presence of a neurodegenerative pathology linked to exposure to traumatic brain injury, chronic traumatic encephalopathy (CTE) neuropathologic change, ${ }^{34}$ which is thought to be associated with neuropsychiatric presentations, such as depression, impulsivity, aggression and suicide. ${ }^{5-7}$ Nevertheless, despite these observations, there remain limited robust epidemiological data regarding mental health outcomes in former contact sports athletes with known risk of neurodegenerative disease.

Early in the 20th century, descriptions of the punch-drunk syndrome of former boxers ${ }^{8}$ led to recognition of the association between exposure to traumatic brain injury and increased risk of neurodegenerative disease. Later described as dementia pugilistica, the clinical picture that emerged was one of a relatively stereotyped syndrome featuring emotional lability, personality change, cognitive impairment and dementia. ${ }^{9}$ This issue came to wider attention through recognition of the pathology of dementia pugilistica, now termed CTE neuropathologic change, ${ }^{10}$ in autopsy studies of wider non-boxer athletes, including former soccer players ${ }^{10} 11$ and others exposed to traumatic brain injury. ${ }^{42-14}$ Notably, in these more modern reports of non-boxer athletes with CTE, in addition to the long-recognised symptoms described in former boxers, reports of behavioural and neuropsychiatric symptomatology have emerged, including aggression, depression and suicidality. ${ }^{5-7}$ Conflicting with these autopsy observations, limited epidemiological studies report lower suicide rates among former National Football League (NFL) American footballers than anticipated from general population data. ${ }^{15} 16$

Played in over 200 countries, soccer is the world's most popular participation sport, with over a quarter of a billion active participants worldwide. Among former professional soccer players neurodegenerative disease mortality is approximately 3.5fold higher than anticipated, with this risk ranging from an approximately doubling of deaths with Parkinson's disease to a fivefold increase in deaths with Alzheimer's disease. ${ }^{17}$ Furthermore, CTE neuropathologic change is present in the majority of former soccer players dying with dementia. ${ }^{10}$ However, data are limited and inconsistent regarding mental health disorders in professional soccer players, with reported rates ranging from higher than expected ${ }^{1819}$ to no different from the general population. ${ }^{20}$

This retrospective cohort study is designed to test the hypothesis that a population of former professional soccer players with known high neurodegenerative mortality are at higher risk of common mental health disorders and have higher rates of suicide than the general population. 
Second, that risk of mental health disorder is associated with player position.

\section{METHODS}

\section{Approvals}

Individual participant-level consent was not required as all health records data were anonymised to the researchers. The protocol for 'Football's InfluencE on Lifelong outcomes and Dementia risk (FIELD)' is published elsewhere. ${ }^{21}$ This study is reported in accordance with the Strengthening the Reporting of Observational Studies in Epidemiology statement. ${ }^{22}$

\section{Cohort identification}

Former professional soccer players were identified from the Record of Pre-war Scottish League Players (V.2) ${ }^{23}$ and the Record of Post-War Scottish League Players (V.6). ${ }^{24}$ These databases are compiled from the archives of the Scottish Football Museum and individual clubs and contain information on all professional Scottish league players, including player demographics (name and date of birth) and career information (dates of first signing and retirement, number of match appearances and player position). From these databases, duplicate entries were merged to a single player record. Probabilistic matching was then used to match former players to their unique Community Health Index, an identifier that links each person in Scotland to their individual electronic health records. Study entry was restricted to those born prior to 1 January 1977. All former soccer players were male. Full details of the cohort are published elsewhere. ${ }^{1721}$

\section{Matched population comparison group}

The Community Health Index database was used to randomly identify a general population comparison group, individually matched by sex, year of birth and social deprivation status to former soccer players on a 3:1 basis; that is, for each former soccer player, three matched population controls were identified. The National Health Service Information Services Division records last known postcode of residence for all individuals, with degree of socioeconomic deprivation for each postcode available as the Scottish Index of Multiple Deprivation (SIMD), which is derived from data on income, employment, health, education, housing and crime. The SIMD is categorised into general population quintiles ranging from 1 (most deprived) to 5 (least deprived).

\section{Outcomes}

Outcomes for former soccer players and the matched population comparison group were obtained by individual-level record linkage to inpatient and day case admissions, held within Scottish Morbidity Record SMR01 (General/Acute Inpatient and Day Case) and Scottish Morbidity Record SMR04 (Mental Health Inpatient and Day Case) datasets and to death certificates. For each dataset, diagnoses are coded using the International Classification of Diseases (ICD-9/ICD-10). The SMR01 and SMR04 datasets were interrogated to identify admissions where a diagnosis was recorded in the principal position as one of the five most common mental health disorders among adult Scottish males, namely anxiety and stress related disorders, depression, alcohol use disorders, drug use disorders, or bipolar and affective mood disorders. Death certification datasets were interrogated for records where suicide was recorded as the cause of death. The relevant ICD-9/ICD-10 codes for these diagnoses are listed in table 1 . Hospital admission and death certification
Table 1 International Classification of Diseases codes included in dataset searches

\begin{tabular}{|c|c|c|}
\hline & ICD-9 & ICD-10 \\
\hline \multicolumn{3}{|l|}{ Mental health disorder } \\
\hline Anxiety and stress related & $\begin{array}{l}300.0-300.9 ; 308.0-308.9 ; \\
309.3-309.9 ; 313.0\end{array}$ & F40-F45; F48; F50; F52 \\
\hline Depression & $296.2-296.3$ & F32-F33 \\
\hline Alcohol use & 291.0-291.9, 303.9 & F10 \\
\hline Drug use & $\begin{array}{l}292.0-292.9,304.0-304.9, \\
305.0-305.9\end{array}$ & F11-F19 \\
\hline $\begin{array}{l}\text { Bipolar and affective } \\
\text { mood disorders }\end{array}$ & 296.0-296.1, 296.4-296.9 & F30-F31, F34-F39 \\
\hline \multicolumn{3}{|l|}{ Mortality } \\
\hline \multirow[t]{3}{*}{ Suicide } & E950-E959 & $X 60-X 84$ \\
\hline & $905-909$ & R458 \\
\hline & $940-949$ & Z915 \\
\hline
\end{tabular}

ICD, International Classification of Diseases.

data were censored on 31 December, 2016, with the database interrogation performed on 10 December 2018.

\section{Statistical analyses}

For the whole cohort, Cox stratified proportional hazard regression was used, and the assumption of proportionality was tested using Schoenfeld residuals. ${ }^{25}$ Age was used as the time covariate, with follow-up until the first eligible event (hospital admission for mental health disorder or suicide respectively) censored for all-cause death or end of follow-up, and the results reported as HRs and 95\% CIs. We repeated the analyses in the former soccer player subgroup using standard Cox proportional hazards with player position (outfield or goalkeeper) as the exposure of interest. Mean age at first eligible event (mental health admission or death from suicide) was calculated using linear regression with cluster robust SEs to account for the case matched nature of the data. All statistical analyses were undertaken using Stata V. $16^{26}$ with statistical significance set at two sided $\mathrm{p}<0.05$.

\section{RESULTS}

Cohort characteristics and common mental health disorders in former soccer players

Over a median of 18 years of follow-up, 388 (5.1\%) of 7676 former professional soccer players and 1399 (6.1\%) of 23028 matched population controls were admitted to hospital for management of a mental health disorder (table 2). Compared with the population comparison group, former professional soccer players had a lower risk of hospital admission for anxiety and stress related disorders (HR 0.37 ; $95 \%$ CI 0.25 to 0.55 ; $\mathrm{p}<0.001$ ), depression (HR $0.64 ; 95 \%$ CI 0.44 to $0.92 ; \mathrm{p}=0.02$ ), drug use disorders (HR 0.39; 95\% CI 0.25 to $0.60 ; \mathrm{p}<0.001$ ), alcohol use disorders (HR 0.62; 95\% CI 0.51 to 0.76 ; $<<0.001$ ) and bipolar and affective mood disorders (HR 0.55; 95\% CI 0.34 to $0.88 ; p=0.01$ ) (table 3 ), with each outcome fulfilling the assumption of proportional hazards.

\section{Age at first admission with mental health disorder and influence of player position}

Age at first admission for depression was higher in former professional soccer players than controls (mean 52.3 \pm 13.6 years vs $46.8 \pm 14.7$ years; $p=0.03$ ). There was no significant difference in age at first admission for the remaining mental health disorders (figure 1). Within the former professional soccer player subgroup, there was no significant difference in the risk of 


\begin{tabular}{|c|c|c|}
\hline & $\begin{array}{l}\text { Former professional } \\
\text { soccer players } n=7676 \\
n(\%)\end{array}$ & $\begin{array}{l}\text { Matched population } \\
\text { controls } n=23028 \\
n(\%)\end{array}$ \\
\hline $\begin{array}{l}\text { Hospital admission with } \\
\text { mental health diagnosis }\end{array}$ & $388(5.1)$ & $1399(6.1)$ \\
\hline \multicolumn{3}{|l|}{ SIMD quintile } \\
\hline 1 (high deprivation) & $1206(15.7)$ & $3617(15.7)$ \\
\hline 2 & $1371(17.9)$ & $4112(17.9)$ \\
\hline 3 & 1449 (18.9) & 4349 (18.9) \\
\hline 4 & $1613(21.0)$ & $4839(21.0)$ \\
\hline 5 (low deprivation) & $2037(26.5)$ & $6111(26.5)$ \\
\hline \multicolumn{3}{|l|}{ Player position } \\
\hline Goalkeeper & $598(7.8)$ & NA \\
\hline Outfield & $6024(78.5)$ & NA \\
\hline Unknown & $1054(13.7)$ & NA \\
\hline
\end{tabular}

NA, not applicable; SIMD, Scottish Index of Multiple Deprivation.

admission for common mental health disorders between former goalkeepers and former outfield players (HR 1.43; 95\% CI 0.92 to $2.23 ; \mathrm{p}=0.11$ ).

\section{Suicide in former professional soccer players}

Over the period of follow-up, there were 1180 (15.4\%) deaths among the former professional soccer players and 3807 (16.5\%) among the population comparison group. Suicide was recorded as the cause of death in $19(0.25 \%)$ of the 7676 former professional soccer players and $93(0.40 \%)$ of the 23028 population controls. There was no significant difference in risk of suicide (HR $0.69 ; 95 \%$ CI 0.25 to $1.87 ; \mathrm{p}=0.47$ ) or age at suicide (mean $44.6 \pm 14.3$ years vs $44.1 \pm 11.7$ years; $p=0.89$ ) between soccer players and their matched controls.

\section{DISCUSSION}

Our data provide evidence that, compared with a general population comparison group matched for age, sex and degree of social deprivation, former professional soccer players were at lower risk of hospital admission for the most common mental health disorders affecting the Scottish male population. Specifically, former soccer players were approximately half as likely to be admitted for anxiety and stress related disorders, depressive disorder, alcohol use disorders, drug use disorders, and bipolar and affective mood disorders. Furthermore, subgroup analysis showed no significant difference in hospital admissions for common mental health disorder between outfield players and goalkeepers. Finally, we found no significant difference in deaths due to suicide between former soccer players and matched population controls.

Autopsy studies on a wide range of former contact sports athletes, including former soccer players, reveal CTE

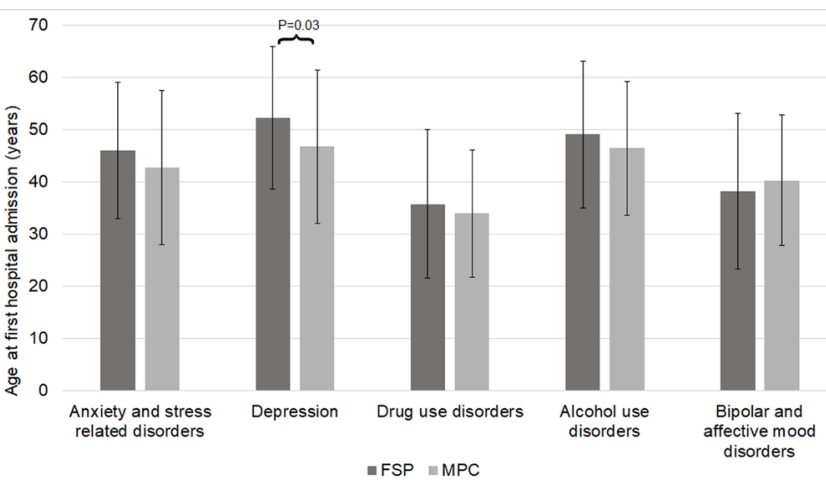

Figure 1 Age at first admission with mental health disorder. Age at first admission with depression was higher in former professional soccer players (FSPs) than matched population controls (MPC) (52.3 \pm 13.6 (mean \pm SD) years vs $46.8 \pm 14.7$ years; $p=0.03$ ). Otherwise, age at first admission for the remaining mental health disorders was the same for former soccer players and matched general population controls.

neuropathologic change in a majority, ${ }^{4} 1013$ either as the primary dementia associated pathology or as a comorbid pathology in context of an alternate neurodegenerative disease. ${ }^{10}$ Alongside this, epidemiological studies describe higher neurodegenerative disease mortality than anticipated among former professional soccer players ${ }^{17}$ and former NFL American footballers. ${ }^{27}$ As such, there are multiple levels of evidence in support of the association between contact sports participation, neurodegenerative pathology, in particular CTE, and clinically confirmed neurodegenerative disease.

In contrast to the long acknowledged cognitive outcomes associated with contact sports participation, symptoms of common mental disorders and suicide were not characteristic of the earliest accounts of former boxers with CTE. ${ }^{9}$ However, more recent reporting of non-boxer athletes has placed greater emphasis on psychiatric symptomatology in CTE. Nevertheless, data supporting this association are weak. Regarding suicide, autopsy studies of donated brains from former NFL American footballers document a number who died by suicide and were subsequently confirmed to have CTE neuropathologic change at autopsy. ${ }^{3} 13$ However, suicide deaths among cases with CTE neuropathologic change were lower than among those without this pathology. ${ }^{3}$ Furthermore, epidemiological data reveal lower rates of suicide in former NFL American footballers than might be expected based on general population datasets. ${ }^{15} 16$ Our observations in former professional soccer players provide further evidence that elite-level contact sport participation is not associated with higher risk of suicide in retirement, despite the documented high neurodegenerative mortality in this population.

At present, no consensus criteria for the clinical diagnosis of CTE exist; the diagnosis requiring autopsy examination with

Table 3 Mental health admissions in former professional soccer players and matched population controls

\begin{tabular}{|c|c|c|c|c|}
\hline Mental health disorder & $\begin{array}{l}\text { Former professional soccer players } \\
\mathrm{n}(\%)\end{array}$ & $\begin{array}{l}\text { Matched population controls } \\
\mathrm{n}(\%)\end{array}$ & HR $(95 \% \mathrm{Cl})$ & P value* \\
\hline Anxiety and stress related disorders & $27(0.35)$ & $222(0.96)$ & $0.37(0.25$ to 0.55$)$ & $<0.001$ \\
\hline Depression & $38(0.50)$ & $169(0.73)$ & $0.64(0.44$ to 0.92$)$ & 0.02 \\
\hline Drug use disorders & $24(0.31)$ & $189(0.82)$ & $0.39(0.25$ to 0.60$)$ & $<0.001$ \\
\hline Alcohol use disorders & $117(1.52)$ & $559(2.43)$ & $0.62(0.51$ to 0.76$)$ & $<0.001$ \\
\hline Bipolar and affective mood disorders & $21(0.27)$ & $112(0.49)$ & $0.55(0.34$ to 0.88$)$ & 0.01 \\
\hline
\end{tabular}

${ }^{*}$ Cox proportional hazard regression. All analyses fulfilled the assumption of proportional hazards. 
formal neuropathological assessment. Nevertheless, research criteria have been proposed to aid identification of the clinical correlate of CTE: traumatic encephalopathy syndrome (TES). These recognise TES on the basis of three core clinical features, of which at least one must be present for a diagnosis to be made. ${ }^{7}$ The proposed core features of TES are cognitive, behavioural (such as emotionally explosive or physically violent) and mood (such as overly sad or depressed). ${ }^{7}$ In addition to these core clinical features, a broad range of supportive features are proposed, which include anxiety, paranoia and suicidality, with at least two supportive features required to diagnose TES. Thus, the currently proposed research criteria for the clinical diagnosis of TES include psychiatric symptoms as both core and supportive features. Contrasting with these criteria for TES diagnosis, our data show no increase in hospital admissions for mental health disorder or in suicides in a population of former professional soccer players with known high neurodegenerative disease mortality. Indeed, we identified lower hospital admissions for common mental health disorder in our former soccer players. As such, the value of psychiatric symptomatology in TES diagnostic criteria require further consideration.

Among the behavioural or mood features proposed as core symptoms of TES are depression, anxiety and mania. ${ }^{7}$ Nevertheless, although a range of mood symptoms are reported in retrospective clinical reviews on patients whose brains have been donated for research, ${ }^{3}$ evidence supporting higher rates of common mental health disorder in former contact sports athletes is limited. Studies on small numbers of former soccer players suggest high prevalence of symptoms of common mental disorder. ${ }^{18}{ }^{19}$ However, issues related to multiple potential recruitment biases, small cohort sizes and absence of matched population control data limit the value of these observations. Accessing a comprehensive database of former professional soccer players and comparing their outcomes to those of population controls matched by age, sex and degree of social deprivation we find former professional soccer players have lower risk of common mental health disorders, in respect of hospital admission data. This finding is in clear contrast to assumptions based on autopsy or survey reporting but might be in line with experience elsewhere reporting the benefits of physical activity on mental health. ${ }^{28}$

Thus far, the only risk factor proposed for CTE remains exposure to traumatic brain injury (TBI) or repetitive 'sub-concussive' head impacts. Purposeful head impacts through football heading are considered part of normal gameplay in soccer. However, although studies report evidence of short-lived cognitive impairment $^{29}$ and elevations in blood biomarkers ${ }^{30}$ acutely after repetitive football heading and evidence of brain structural changes over a season on imaging studies, ${ }^{31}$ the late consequences of cumulative exposure to heading remain unknown. We hypothesised goalkeepers to be a population at lower risk of adverse outcome from exposure to TBI compared with outfield players. In support of this, data demonstrate rates of concussion are considerably lower in goalkeepers compared with outfield players. ${ }^{32}$ Further, we have previously demonstrated lower neurodegenerative disease morbidity in former goalkeepers than outfield players. ${ }^{17}$ In this current study, we found no difference in admissions for common mental health disorders between goalkeepers and outfield players, lending support to the suggestion that diagnoses of neurodegenerative disease and mental health disorder are independent in former professional soccer players. These data also provide no evidence to support an association between exposure to head impacts and adverse mental health outcomes in this population.
One possible confounding factor contributing to our observations is that individuals pursuing a career as an elite athlete may have inherent health differences from the general population, the so called 'healthy worker' effect. ${ }^{33}$ In other words, conceptually, mental health issues might at some level serve as an impediment to pursuit of a career as an elite soccer player. Against this suggestion, available data provide no supportive evidence of lower rates of common mental health disorders in elite athletes compered with general population data. ${ }^{34}$ Thus, elite athletes, including soccer players, have been reported with higher, or similar, rates of depression ${ }^{35}{ }^{36}$ and anxiety ${ }^{36}$ with contradictory data in relation to suicide: higher in elite power athletes ${ }^{37}$ but lower in former professional American footballers. ${ }^{15}$

To our knowledge, this is the first study to access diagnostic datasets to investigate the association between participation in elite level contact sports and risk of common mental health disorders in retirement. By accessing extensive electronic health records on a large cohort of soccer players and their age and social deprivation matched population controls, our study design compensates for factors with recognised impact on mental health outcomes. This study does not, however, capture information on patients managed exclusively in a primary care setting and so not requiring review by hospital services. Mental health disorders managed in the community typically are less significant clinically but more frequent than mental health disorders requiring hospital admission. However, we have no reason to presume that there would be a difference in diagnostic reporting or level of healthcare accessed by former professional soccer players and their matched controls.

\section{Summary}

In summary, when compared with matched general population controls, our data demonstrate that, despite known high neurodegenerative disease mortality, former professional soccer players are at lower risk of hospital admission for the most common mental health disorders among the Scottish male population. Furthermore, deaths as a result of suicide did not differ significantly between former professional soccer players and their matched controls.

\section{Twitter William Stewart @WillStewNeuro}

Contributors ERR and DFM collated the former soccer player and matched population control datasets. ERR completed all the primary data analyses. ERR and TM provided a first draft of the analyses and manuscript. ERR, TM, DFM, KS, JAM, JPP and WS edited manuscript drafts and WS collated all author comments to the final submitted version. ERR, TM, DFM, KS, JAM, JPP and WS contributed to discussions on data analysis and interpretation, commented on manuscript drafts and approved the final version. The corresponding author had full access to all the data in the study and had final responsibility for the decision to submit for publication.

Funding This work was supported by funding from: The Football Association and Professional Footballers Association and an NHS Research Scotland Career Researcher Fellowship (WS).

Competing interests None declared.

Patient consent for publication Not required.

Ethics approval Approval for this study was provided by the University of Glasgow College of Medical, Veterinary and Life Sciences Ethics Committee (Project Number 200160147), with protocol and data governance procedures reviewed and approved by National Health Service Scotland's Public Benefit and Privacy Panel for Health and Social Care (reference 1718-0120).

Provenance and peer review Not commissioned; externally peer reviewed.

Data availability statement Data are available on reasonable request. Per Public Benefits and Privacy Panel (PBPP) authorisations, electronic health records data are stored on secure server with access granted only to specified investigators after approval. Subject to approvals from PBPP datasets should be accessible. 
ORCID ID

William Stewart http://orcid.org/0000-0003-2199-2582

\section{REFERENCES}

1 Smith DH, Johnson VE, Trojanowski JQ, et al. Chronic traumatic encephalopathy confusion and controversies. Nat Rev Neurol 2019;15:179-83.

2 Stewart W, Allinson K, Al-Sarraj S, et al. Primum non nocere: a call for balance when reporting on CTE. Lancet Neurol 2019;18:231-3.

3 McKee AC, Stern RA, Nowinski CJ, et al. The spectrum of disease in chronic traumatic encephalopathy. Brain 2013;136:43-64.

4 Smith DH, Johnson VE, Stewart W. Chronic neuropathologies of single and repetitive TBI: substrates of dementia? Nat Rev Neurol 2013;9:211-21.

5 Omalu BI, Bailes J, Hammers JL, et al. Chronic traumatic encephalopathy, suicides and parasuicides in professional American athletes: the role of the forensic pathologist. Am J Forensic Med Pathol 2010;31:130-2.

6 Stern RA, Daneshvar DH, Baugh CM, et al. Clinical presentation of chronic traumatic encephalopathy. Neurology 2013;81:1122-9.

7 Montenigro PH, Baugh CM, Daneshvar DH, et al. Clinical subtypes of chronic traumatic encephalopathy: literature review and proposed research diagnostic criteria for traumatic encephalopathy syndrome. Alzheimers Res Ther 2014:6:68.

8 Martland HS. Punch drunk. J Am Med Assoc 1928;91:1103-7.

9 Roberts AH. Brain damage in boxers: a study of the prevalence of traumatic encephalopathy among ex-professional boxers. London: Pitman Med. Sci. Publ, 1969.

10 Lee EB, Kinch K, Johnson VE, et al. Chronic traumatic encephalopathy is a common co-morbidity, but less frequent primary dementia in former soccer and rugby players. Acta Neuropathol 2019;138:389-99.

11 Ling H, Morris HR, Neal JW, et al. Mixed pathologies including chronic traumatic encephalopathy account for dementia in retired association football (soccer) players. Acta Neuropathol 2017:133:337-52.

12 Stewart W, McNamara PH, Lawlor B, et al. Chronic traumatic encephalopathy: a potential late and under recognized consequence of rugby union? QJM 2016;109:11-15.

13 Mez J, Daneshvar DH, Kiernan PT, et al. Clinicopathological evaluation of chronic traumatic encephalopathy in players of American football. JAMA 2017;318:360-70.

14 Zanier ER, Bertani I, Sammali E, et al. Induction of a transmissible tau pathology by traumatic brain injury. Brain 2018;141:2685-99.

15 Lehman EJ, Hein MJ, Gersic CM. Suicide mortality among retired National football League players who played 5 or more seasons. Am J Sports Med 2016;44:2486-91.

16 Webner D, Iverson GL. Suicide in professional American football players in the past 95 years. Brain Inj 2016;30:1718-21.

17 Mackay DF, Russell ER, Stewart K, et al. Neurodegenerative disease mortality among former professional soccer players. N Engl J Med 2019;381:1801-8.

18 Gouttebarge V, Aoki H, Lambert M, et al. A history of concussions is associated with symptoms of common mental disorders in former male professional athletes across a range of sports. Phys Sportsmed 2017;45:443-9.
19 Gouttebarge V, Castaldelli-Maia JM, Gorczynski P, et al. Occurrence of mental health symptoms and disorders in current and former elite athletes: a systematic review and meta-analysis. Br J Sports Med 2019;53:700-6.

20 Fernandes GS, Parekh SM, Moses J, et al. Depressive symptoms and the general health of retired professional footballers compared with the general population in the UK: a case-control study. BMJ Open 2019:9:e030056.

21 Russell ER, Stewart K, Mackay DF, et al. Football's InfluencE on Lifelong health and Dementia risk (FIELD): protocol for a retrospective cohort study of former professional footballers. BMJ Open 2019;9:e028654.

22 von Elm E, Altman DG, Egger M, et al. The strengthening the reporting of observational studies in epidemiology (STROBE) statement: guidelines for reporting observational studies. Lancet 2007;370:1453-7.

23 Litster JA. Record of pre-war Scottish League players: version 2. Available: http:// www.pmfc.co.uk/prewar.html [Accessed 22 May 2019].

24 Litster JA. Record of post-war Scottish League players: version 6. Available: http:// www.pmfc.co.uk/postwar.html [Accessed 22 May 2019].

25 Cleves M, Gould W, Gutierrez R, et al. An introduction to survival analysis using Stata. Second Edition. Stata Press, 2008.

26 StataCorp. Stata statistical software: release 16. College Station, TX: StataCorp LLC, 2019.

27 Lehman EJ, Hein MJ, Baron SL, et al. Neurodegenerative causes of death among retired National football League players. Neurology 2012;79:1970-4.

28 Chekroud SR, Gueorguieva R, Zheutlin AB, et al. Association between physical exercise and mental health in 1.2 million individuals in the USA between 2011 and 2015: a cross-sectional study. Lancet Psychiatry 2018;5:739-46.

29 Di Virgilio TG, Hunter A, Wilson L, et al. Evidence for acute electrophysiological and cognitive changes following routine soccer heading. EBioMedicine 2016;13:66-71.

30 Wallace C, Smirl JD, Zetterberg $\mathrm{H}$, et al. Heading in soccer increases serum neurofilament light protein and SCAT3 symptom metrics. BMJ Open Sport Exerc Med 2018;4:e000433.

31 Lipton ML, Kim N, Zimmerman ME, et al. Soccer heading is associated with white matter microstructural and cognitive abnormalities. Radiology 2013;268:850-7.

32 Putukian M, Echemendia RJ, Chiampas G, et al. Head injury in soccer: from science to the field; summary of the head injury Summit held in April 2017 in New York City, New York. Br J Sports Med 2019:53:1332.

$33 \mathrm{Li} \mathrm{CY}$, Sung FC. A review of the healthy worker effect in occupational epidemiology. Occup Med 1999:49:225-9.

34 Reardon CL, Hainline B, Aron CM, et al. Mental health in elite athletes: international Olympic Committee consensus statement (2019). Br J Sports Med 2019:53:667-99.

35 Junge A, Feddermann-Demont N. Prevalence of depression and anxiety in top-level male and female football players. BMJ Open Sport Exerc Med 2016;2:e000087.

36 Gouttebarge V, Jonkers R, Moen M, et al. The prevalence and risk indicators of symptoms of common mental disorders among current and former Dutch elite athletes. J Sports Sci 2017;35:2148-56.

37 Lindqvist A-S, Moberg T, Ehrnborg C, et al. Increased mortality rate and suicide in Swedish former elite male athletes in power sports. Scand I Med Sci Sports 2014;24:1000-5. 